Research Article

\title{
Numerical Investigation of Complex Thermal Coal-Gas Interactions in Coal-Gas Migration
}

\author{
Xiaoyan $\mathrm{Ni}$ (D), ${ }^{1,2}$ Peng Gong $\mathbb{D}^{2},{ }^{2}$ and Yi Xue $\mathbb{i D}^{3}$ \\ ${ }^{1}$ School of Construction Engineering, Jiangsu Vocational Institute of Architectural Technology, Xuzhou 221116, China \\ ${ }^{2}$ State Key Laboratory for Geomechanics and Deep Underground Engineering, China University of Mining and Technology, \\ Xuzhou 221116, China \\ ${ }^{3}$ Institute of Geotechnical Engineering, Shaanxi Provincial Key Laboratory of Loess Mechanics and Engineering, \\ Xi'an University of Technology, Xi'an 710048, China
}

Correspondence should be addressed to Yi Xue; xueyi@xaut.edu.cn

Received 30 August 2018; Accepted 15 November 2018; Published 5 December 2018

Guest Editor: Dengke Wang

Copyright (c) 2018 Xiaoyan Ni et al. This is an open access article distributed under the Creative Commons Attribution License, which permits unrestricted use, distribution, and reproduction in any medium, provided the original work is properly cited.

Understanding the influence of temperature on the gas seepage of coal seams is helpful to achieve the efficient extraction of underground coal seam gas. Thermal coal-gas interactions involve a series of complex interactions between gas and solid coal. Although the interactions between coal and gas have been studied thoroughly, few studies have considered the temperature evolution characteristics of coal seam gas extraction under the condition of variable temperature because of the complexity of the temperature effect on gas drainage. In this study, the fully coupled transient model combines the relationship of gas flow, heat transfer, coal mass deformation, and gas migration under variable temperature conditions and represents an important nonlinear response to gas migration caused by the change of effective stress. Then, this complex model is implemented into a finite element (FE) model and solved through the numerical method. Its reliability was verified by comparing with historical data. Finally, the effect of temperature on coal permeability and gas pressure is studied. The results reveal that the gas pressure in coal fracture is generally higher than that in the matrix blocks. The higher temperature of the coal seam induces the faster increase of the gas pressure. Temperature has a great effect on the gas seepage behavior in the coal seams.

\section{Introduction}

Coal seam gas is an important natural source and an important part in the clean energy structure in China [1]. However, most Chinese coalbed methane cannot meet the requirements of effective recovery because of the low permeability characteristic $[2,3]$. Therefore, many technological means are adopted to enhance the coal permeability and develop the gas extraction efficiency $[4,5]$, such as, hydraulic fracturing technology [6]. Besides, hot injection has been adopted to enhance the extraction of coalbed methane $[7,8]$. Therefore, the thermal evolution characteristic evolution during the extraction of coalbed methane needs to be studied.

The thermal stimulation of gas reservoir triggers the complex interaction between coal, gas, and temperature. It changes the deformation behavior of coal, as well as the heat and gas flow. Temperature has an obvious effect on the adsorption capacity of coal seam gas, which is negatively correlated with the increase of temperature [9-11]. At the same time, the thermal expansion coefficient is heterogeneous and anisotropic due to the different response of different components to temperature changes. Noorishad et al. [12] evaluated the coupled thermal-hydraulicmechanical characteristics of rock and investigated the effects of thermal stresses on permeability through the deformation change of the fractures. Mctigue [13] developed a fluid-saturated, porous, thermoelastic model and calculated the heating of a half space at constant temperature conditions. Harpalani and Schraufnagel [14] found that desorption induced the shrinking of the coal matrix and increased permeability through seepage tests. Zhou et al. [15] 
consider thermodynamically coupled water and heat flow and demonstrate the influence of thermodynamic coupling through numerical and analytical solutions. Zhu et al. [16] analyzed the influence of temperature on coal permeability based on a complex thermal-hydrological-mechanical model and found that sorption-induced volumetric strain affected the seepage behavior of coal seam significantly. Cai et al. [17] found that thermal treatment can promote methane desorption and increase the permeability through the numerical simulation. Qu et al. [18] developed a new thermal model with gas flow and matrix adsorption and suggested that the increase in temperature leads to the decrease in coal swelling and larger cleat aperture and higher coal permeability.

The interaction mechanism of heat injection has not yet fully understood for the design of engineering. It is indispensable to study the coal-gas interaction under variable temperature. In this study, a coupled thermal-hydrologicalmechanical model is established, considering the sorption characteristic and permeability evolution. Then, this complex model is implemented into a finite element (FE) and solved through the numerical method. Finally, the effect of temperature on coal permeability and gas pressure is studied based on this coupled model.

\section{Equations of Coupled Model}

2.1. Gas Seepage Characteristic in Coal Fractures. For the coal seam reservoir, the non-Darcy flow can be expressed as

$$
-\nabla p_{\mathrm{f}}=\frac{v}{k_{\mathrm{g}}} \vec{\mu}+\beta \rho_{\mathrm{g}} \vec{\mu}|\vec{\mu}|,
$$

where $\vec{\mu}$ is velocity vector, $\rho_{\mathrm{g}}$ is density, $\beta=1.75 / \sqrt{150 k_{\mathrm{g}} \phi^{3}}$, $\phi$ is porosity, and $k_{\mathrm{g}}$ is the permeability of coal. form:

The above equation can be expressed in the following

$$
\vec{\mu}=-\frac{k_{\mathrm{g}}}{\left(1+\left(k_{\mathrm{g}} / v\right) \beta \rho_{\mathrm{g}}|\vec{\mu}|\right) v} \nabla p_{\mathrm{f}}=-\frac{k_{\mathrm{g}}}{f_{\mathrm{q} i} v} \nabla p_{\mathrm{f}},
$$

where $f_{\mathrm{qi}}=1+\left(k_{\mathrm{g}} / v\right) \beta \rho_{\mathrm{g}}|\vec{\mu}|$ is the Forchheimer coefficient.

The mass conservation equation of gas migration can be expressed as

$$
\frac{\partial}{\partial t}\left(\phi_{\mathrm{f}} \rho_{\mathrm{g}}\right)+\nabla\left(\rho_{\mathrm{g}} \cdot \vec{\mu}\right)=Q_{\mathrm{s}}\left(1-\phi_{\mathrm{f}}\right)
$$

where $\vec{\mu}$ is the velocity vector and $Q_{s}$ is the gas source, and the mass content can be expressed as [19]

$$
m=\rho_{\mathrm{g}} \phi_{\mathrm{f}}+\rho_{\mathrm{ga}} \rho_{\mathrm{c}} V_{\mathrm{sg}},
$$

where $\phi_{\mathrm{f}}$ is the porosity, $\rho_{\mathrm{ga}}$ is the gas density, $\rho_{\mathrm{c}}$ is the coal density, and $V_{\mathrm{sg}}$ is the absorbed content.

In the coal seams, the gas absorption volume can be expressed as

$$
V_{\mathrm{sg}}=\frac{V_{\mathrm{L}} p_{\mathrm{f}}}{p_{\mathrm{f}}+P_{\mathrm{L}}} \exp \left[-\frac{c_{2}}{1+c_{1} p_{\mathrm{f}}}\left(T_{\mathrm{ar}}+T-T_{\mathrm{t}}\right)\right] .
$$

The Sorption Strain $\varepsilon_{s}$ can be expressed as

$$
\varepsilon_{\mathrm{s}}=\alpha_{\mathrm{sg}} V_{\mathrm{sg}},
$$

where $V_{\mathrm{sg}}$ is the content of absorbed gas and $\alpha_{\mathrm{sg}}$ is the strain coefficient.

The gas density can be obtained as

$$
\rho_{\mathrm{g}}=\frac{M_{\mathrm{g}}}{R\left(T_{\mathrm{ar}}+T\right)} p
$$

The mass conservation equation can be expressed as

$$
\frac{\rho_{\mathrm{ga}}}{p_{\mathrm{a}}} \frac{\partial\left(\phi_{\mathrm{f}} p_{\mathrm{f}}\right)}{\partial t}+\nabla\left(-\frac{k_{\mathrm{g}}}{\mu} \frac{\rho_{\mathrm{g}}}{f_{\mathrm{qi}}} \nabla p_{\mathrm{f}}\right)=Q_{\mathrm{s}}\left(1-\phi_{\mathrm{f}}\right) .
$$

2.2. Gas Diffusion in Coal Matrix. The gas migration process experiences three substeps: flow in fracture, gas diffusion, and sorption in matrix [20]. Figure 1 shows a conceptual model for gas transport. The source term can be expressed as

$$
\begin{aligned}
Q_{\mathrm{s}} & =D \sigma_{\mathrm{c}}\left(c_{\mathrm{m}}-c_{\mathrm{f}}\right), \\
\frac{\partial m_{\mathrm{m}}}{\partial t} & =-\frac{\mathrm{Mc}}{\tau R T}\left(p_{\mathrm{m}}-p_{\mathrm{f}}\right),
\end{aligned}
$$

where $Q_{s}$ is the exchange from matrix to fractures, $c_{\mathrm{m}}$ is the gas concentration in matrix, $c_{\mathrm{f}}$ is the gas concentration, $\sigma_{\mathrm{c}}$ is the shape factor, and $\tau$ is the sorption time.

The relationship of gas concentration and gas pressure is written as

$$
\begin{gathered}
c_{\mathrm{m}}=\frac{\mathrm{Mc}}{R T} p_{\mathrm{m}}, \\
c_{\mathrm{f}}=\frac{\mathrm{Mc}}{R T} p_{\mathrm{f}},
\end{gathered}
$$

where $\mathrm{Mc}$ is the molar mass.

The diffusion equation can be written as

$$
\frac{\mathrm{d} m_{\mathrm{b}}}{\mathrm{d} t}=-\frac{1}{\tau}\left(m_{\mathrm{b}}-m_{\mathrm{e}}\right)
$$

where $m_{\mathrm{e}}$ is the equilibrium gas content.

Then, the diffusion equation can be expressed as

$$
\frac{\partial m_{\mathrm{m}}}{\partial t}=-\frac{\mathrm{Mc}}{\tau R T}\left(p_{\mathrm{m}}-p_{\mathrm{f}}\right)
$$

The permeability $k_{\mathrm{g}}$ can be written as [21]

$$
k_{\mathrm{g}}=k_{\infty}\left(1+\frac{b}{p_{\mathrm{f}}}\right)
$$

where $b$ is the Klinkenberg coefficient, $b=\alpha_{\mathrm{k}} k_{\infty}^{-0.36}$, where $\alpha_{\mathrm{k}}$ is the Klinkenberg effect coefficient, $\alpha_{\mathrm{k}}=0.251$.

2.3. Solid Mechanics Equation. The stress equation of coal seam can be written as

$$
\sigma_{\mathrm{eij}}=\sigma_{\mathrm{ij}}-\alpha p_{\mathrm{f}} \delta_{\mathrm{ij}} .
$$




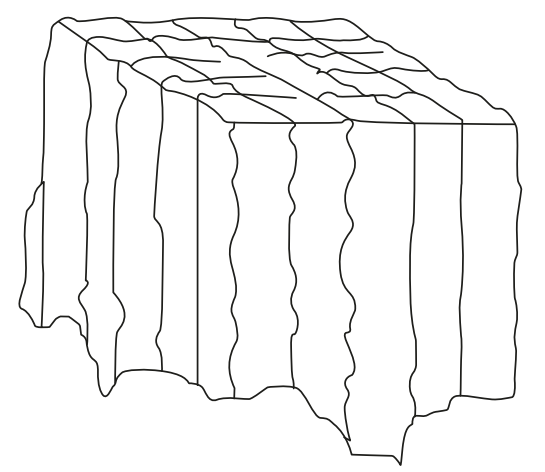

(a)

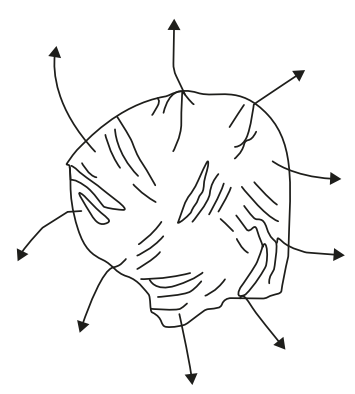

(b)

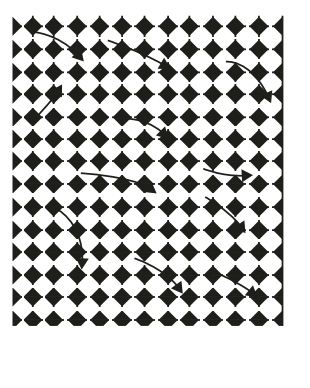

(c)

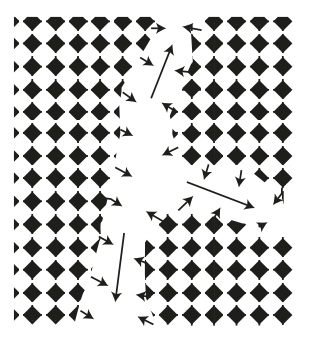

(d)

Figure 1: A conceptual model for gas storage and transport in the coal seams. (a) Natural fracture networks. (b) Stage 1: desorption from internal surfaces. (c) Stage 2: flow/diffusion in the matrix. (d) Stage 3: flow in the natural fracture network.

The stress-strain equation can be written as

$$
\varepsilon_{\mathrm{ij}}=\frac{1}{2}\left(u_{\mathrm{i}, \mathrm{j}}+u_{\mathrm{j}, \mathrm{i}}\right)
$$

The overall stress balance equation can be written as

$$
G u_{\mathrm{i}, \mathrm{jj}}+\frac{G}{1-2 \mu} u_{\mathrm{j}, \mathrm{ji}}-\alpha p_{\mathrm{f}, \mathrm{i}}-K \alpha_{\mathrm{T}} T_{\mathrm{i}}-K \varepsilon_{\mathrm{s}, \mathrm{i}}+f_{\mathrm{i}}=0
$$

2.4. Coal Permeability. The relationship between porosity and effective stress can be expressed as [22]

$$
\Delta \phi_{\mathrm{f}}=\frac{1}{K}\left(\beta_{\mathrm{f}}-\phi_{\mathrm{f}}\right)\left(\bar{\sigma}+p_{\mathrm{f}}\right) .
$$

Then, the porosity is expressed as

$$
\phi_{\mathrm{f}}=\alpha-\left(\alpha-\phi_{0}\right) \exp \left\{-\frac{1}{K}\left[\left(\bar{\sigma}-\bar{\sigma}_{0}\right)+\left(p_{\mathrm{f}}-p_{\mathrm{f} 0}\right)\right]\right\} \text {. }
$$

By substituting the porosity, it can be rewritten as

$$
\begin{aligned}
\phi_{\mathrm{f}}= & \alpha-\left(\alpha-\phi_{\mathrm{f} 0}\right) \exp \left\{-\left[\left(\varepsilon_{\mathrm{V}}+\frac{p_{\mathrm{f}}}{K_{\mathrm{s}}}-\varepsilon_{\mathrm{s}}-\alpha_{\mathrm{T}} T\right)\right.\right. \\
& \left.\left.-\left(\varepsilon_{\mathrm{V} 0}+\frac{p_{\mathrm{f} 0}}{K_{\mathrm{s}}}-\varepsilon_{\mathrm{s} 0}-\alpha_{\mathrm{T}} T_{0}\right)\right]\right\},
\end{aligned}
$$

where $S=\varepsilon_{\mathrm{V}}+\left(p_{\mathrm{f}} / K_{\mathrm{s}}\right)-\varepsilon_{\mathrm{s}}$ and $S_{0}=\varepsilon_{\mathrm{V} 0}+\left(p_{\mathrm{f} 0} / K_{\mathrm{s}}\right)-\varepsilon_{\mathrm{s} 0} \cdot p_{0}$ is the initial pressure, and $\phi_{0}$ is the initial porosity.

The evolution characteristics of permeability can be expressed as

$$
\frac{k_{\mathrm{g}}}{k_{\mathrm{\infty} 0}}=\frac{k_{\infty}}{k_{\infty 0}}\left(1+\frac{b}{p_{\mathrm{f}}}\right)=\left(\frac{\phi}{\phi_{0}}\right)^{3}\left(1+\frac{b}{p_{\mathrm{f}}}\right) .
$$

2.5. Energy Conservation. Total heat flux $q_{\mathrm{T}}$ is expressed as

$$
q_{\mathrm{T}}=-\lambda_{\mathrm{M}} \nabla T+\rho_{\mathrm{g}} C_{\mathrm{g}} q_{\mathrm{g}}\left(T_{\mathrm{ar}}+T\right),
$$

where $q_{\mathrm{T}}$ is the thermal flux and $\rho_{\mathrm{s}}$ is the mass density.

The thermal balance can be written as [23]

$$
\begin{aligned}
& \frac{\partial\left[(\rho C)_{\mathrm{M}}\left(T_{\mathrm{ar}}+T\right)\right]}{\partial t}+\left(T_{\mathrm{ar}}+T\right) K_{\mathrm{g}} \alpha_{\mathrm{g}} \nabla \\
& \cdot\left(\frac{k_{\mathrm{g}}}{\mu} \nabla p\right)+\left(T_{\mathrm{ar}}+T\right) K \alpha_{\mathrm{T}} \frac{\partial \varepsilon_{\mathrm{V}}}{\partial t}=-\nabla \cdot q_{\mathrm{T}},
\end{aligned}
$$

where $(\rho C)_{\mathrm{M}}=\phi_{\mathrm{f}}\left(\rho_{\mathrm{g}} C_{\mathrm{g}}\right)+\left(1-\phi_{\mathrm{f}}\right)\left(\rho_{\mathrm{s}} C_{\mathrm{s}}\right)$ and $\rho_{\mathrm{s}}$ is the mass density.

The conservation of mass yields

$$
\begin{aligned}
\frac{\partial\left[\left(1-\phi_{f}\right) \rho_{\mathrm{s}}\right]}{\partial t} & =0, \\
\frac{\partial\left(\phi_{\mathrm{f}} \rho_{\mathrm{g}}\right)}{\partial t} & =-\nabla \cdot\left(\rho_{\mathrm{g}} q_{\mathrm{g}}\right) .
\end{aligned}
$$

Considering $(1-\phi) \lambda_{\mathrm{s}} \gg \phi \lambda_{\mathrm{s}}$ and $\lambda_{\mathrm{M}} \approx\left(1-\phi_{\mathrm{f}}\right) \lambda_{\mathrm{s}} \approx \lambda_{\mathrm{s}}$, it yields [16]

$$
\begin{aligned}
& (\rho C)_{\mathrm{M}} \frac{\partial T}{\partial t}-\left(T_{\mathrm{ar}}+T\right) K_{\mathrm{g}} \alpha_{\mathrm{g}} \nabla \cdot\left(\frac{k_{\mathrm{g}}}{v} \nabla p_{\mathrm{f}}\right)+\left(T_{\mathrm{ar}}+T\right) K \alpha_{\mathrm{T}} \frac{\partial \varepsilon_{\mathrm{V}}}{\partial t} \\
& =\lambda_{\mathrm{M}} \nabla^{2} T+\frac{\rho_{\mathrm{ga}} p_{\mathrm{f}} T_{\mathrm{a}} C_{\mathrm{g}}}{p_{\mathrm{a}}\left(T_{\mathrm{ar}}+T\right)} \frac{k_{\mathrm{g}}}{v} \nabla p_{\mathrm{f}} \nabla T .
\end{aligned}
$$

These equations (8), (12), (16), and (24) describe the fully coupling model of coal seam gas migration, including mechanical deformation of coal (gas desorption induces coal shrinkage and self-heat induces coal expansion), gas diffusion from the matrix, gas flow, and heat transfer in fractures. The coupled relationship is illustrated in Figure 2. The THM model indicates the nonlinear response of gas migration in underground coal seams. It is hard to get an analytic solution of these equations, so they are achieved through the numerical method with Comsol Multiphysics (a powerful partial differential equation solver) and appropriate boundary conditions.

\section{Model Establishment and Analysis}

3.1. Verification with Field Test. To verify the validity of the model established in this paper in the calculation of 


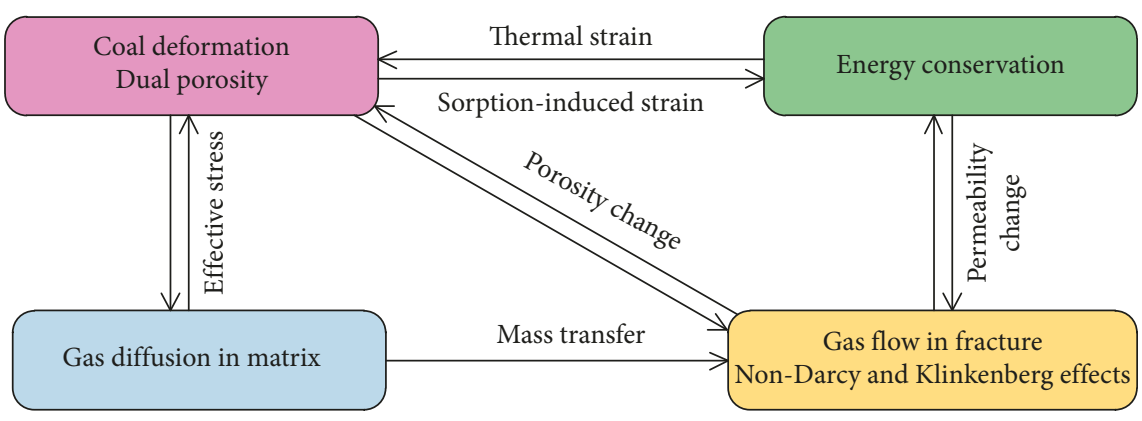

FIgURE 2: Couplings in the gas migration process.

simulated extraction, the numerical simulation is carried out according to the practical example of coal seam extraction. The actual size of the coal seam is $568 \times 568 \mathrm{~m}^{2}$, and the parameters are obtained from Mora and Wattenbarger [24]. The numerical results and the field production data are compared in Figure 3. Good agreement was achieved between the results of numerical simulation and the field production data, which proves the validity of the model.

3.2. Model Establishment. A model is established to analyze the effect of adsorption on the distribution of coal permeability and gas pressure. The size of the model is $0.1 \mathrm{~m} \times$ $0.1 \mathrm{~m}$, as shown in Figure 4. The upper boundary is free. And the condition of the upper boundary is the constant gas pressure $2.5 \mathrm{MPa}$ and the constant temperature $333 \mathrm{~K}$. The initial pressure of the coal seam is $0.1 \mathrm{MPa}$ and the parameters in the calculation are listed in Table 1.

3.3. Effect of Adsorption on Gas Pressure. There are two different pressures between matrix blocks and coal fractures. Because of the high permeability in coal fractures, gas can quickly flow through the fractures. The gas pressure in fractures is generally higher than that in the matrix blocks in the calculation model. The matrix also continues to adsorb gas, and the pressure rises gradually. As the gas is injected from the top of the model, the crack pressure reaches $2.5 \mathrm{MPa}$ firstly at the upper boundary, and the matrix permeability is also increasing. Finally, pore pressure and matrix pressure reach the equilibrium state. Figure 5 shows the gas pressure distribution at different times. As the time goes on, the difference of pressure becomes smaller and smaller. The initial pressure in the coal seam is $0.1 \mathrm{MPa}$. The gas pressure maximums of coal matrix are $0.3 \mathrm{MPa}, 1.8 \mathrm{MPa}$, and $2.3 \mathrm{MPa}$ at $1 \mathrm{~h}, 4 \mathrm{~h}$, and $6 \mathrm{~h}$, respectively. Finally, two pressures reach $2.5 \mathrm{MPa}$ at about 24 hours.

Figure 6 shows the gas pressure distribution at the monitoring line. The difference between the matrix pressure and the fracture pressure can be seen more easily from the detection line. The gas pressure at the bottom of the model is low, while the gas pressure at the entrance is high. Within the equilibrium time of $1 \mathrm{~h}, 4 \mathrm{~h}, 8 \mathrm{~h}, 12 \mathrm{~h}$, and $24 \mathrm{~h}$, the minimum pressure of the coal fracture is $0.1 \mathrm{MPa}, 1.42 \mathrm{MPa}, 2.16 \mathrm{MPa}$, 2.39 MPa, and 2.5 $\mathrm{MPa}$, respectively; meanwhile, the minimum pressure of the coal matrix is $0.1 \mathrm{MPa}, 0.64 \mathrm{MPa}$, 1.66 MPa, 2.19 $\mathrm{MPa}$, and $2.5 \mathrm{MPa}$. With the passage of time,

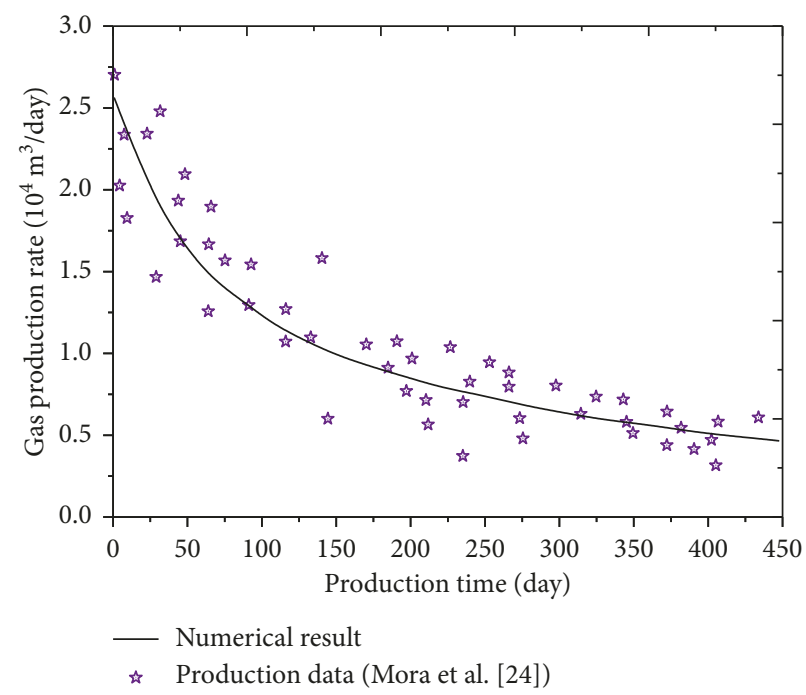

Figure 3: Comparison between numerical result and field test.

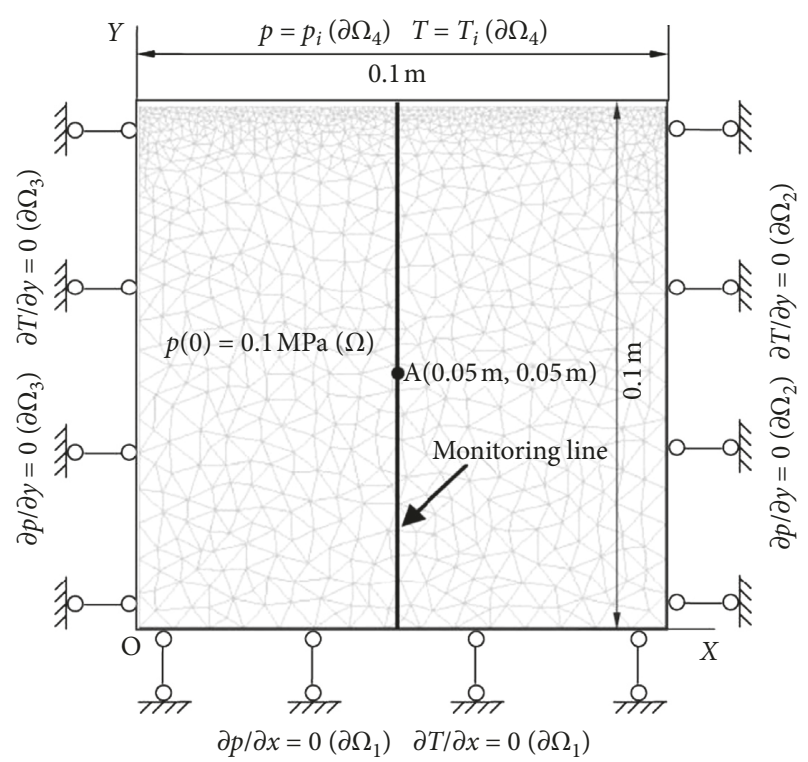

Figure 4: Calculation model.

the pressure of internal coal mass increases gradually. The ratios of minimum matrix pressure and the minimum fracture pressure are $45 \%, 76 \%$, and $92 \%$ at 4 hours, 8 hours, and 12 hours, respectively. 
TABLE 1: Parameters of the model.

\begin{tabular}{|c|c|c|c|c|c|}
\hline Parameter & Value & Sources & Parameter & Value & Sources \\
\hline Young's modulus of coal, $E(\mathrm{MPa})$ & 2713 & {$[22]$} & $\mathrm{CH}_{4}$ Langmuir volume constant, $V_{\mathrm{L}}\left(\mathrm{m}^{3} / \mathrm{kg}\right)$ & 0.043 & [22] \\
\hline Young's modulus of the coal grains, $E_{\mathrm{s}}(\mathrm{MPa})$ & 4070 & {$[22]$} & Specific heat capacity of gas, $C_{\mathrm{g}}(\mathrm{J} / \mathrm{kg} \cdot \mathrm{K})$ & $1.625 \times 10^{3}$ & {$[16]$} \\
\hline Initial porosity, $\phi_{0}(-)$ & 0.01 & [22] & Specific heat capacity of coal, $C_{\mathrm{s}}(\mathrm{J} / \mathrm{kg} \cdot \mathrm{K})$ & $1.25 \times 10^{3}$ & {$[16]$} \\
\hline$\rho_{c}\left(\mathrm{~kg} / \mathrm{m}^{3}\right)$ & 1250 & {$[22]$} & nt, $c_{1}\left(\mathrm{MPa}^{-1}\right)$ & 0.07 & [16] \\
\hline of coal, $v(-)$ & 0.34 & {$[22]$} & Sorption strain coefficient, $\alpha_{\mathrm{sg}}\left(\mathrm{kg} / \mathrm{m}^{3}\right)$ & 0.06 & {$[18]$} \\
\hline Initial gas permeability, $k_{\infty 0}\left(\mathrm{~m}^{2}\right)$ & $1.09 \times 10^{-18}$ & {$[22]$} & Volumetric coefficient of matrix, $\alpha_{\mathrm{T}}\left(\mathrm{K}^{-1}\right)$ & $2.4 \times 10^{-5}$ & {$[18]$} \\
\hline $\begin{array}{l}\text { Density of } \mathrm{CH}_{4} \text { at standard condition, } \rho_{\mathrm{ga}} \\
\left(\mathrm{kg} / \mathrm{m}^{3}\right)\end{array}$ & 0.717 & [1 & Klinkenberg effect, $l$ & & [18] \\
\hline Gas dynamic viscosity, $v\left(\mathrm{~N} \cdot \mathrm{s} / \mathrm{m}^{2}\right)$ & 84 & {$[1$} & $x^{-1}$ & 0.02 & [16] \\
\hline $\mathrm{CH}_{4}$ Langmuir pressure constant, $P_{\mathrm{L}}(\mathrm{MPa})$ & 1.57 & [16] & Thermal conductivity of coal, $\lambda_{\mathrm{s}}(\mathrm{J} /(\mathrm{m} \cdot \mathrm{s} \cdot \mathrm{K}))$ & 0.2 & [16] \\
\hline
\end{tabular}
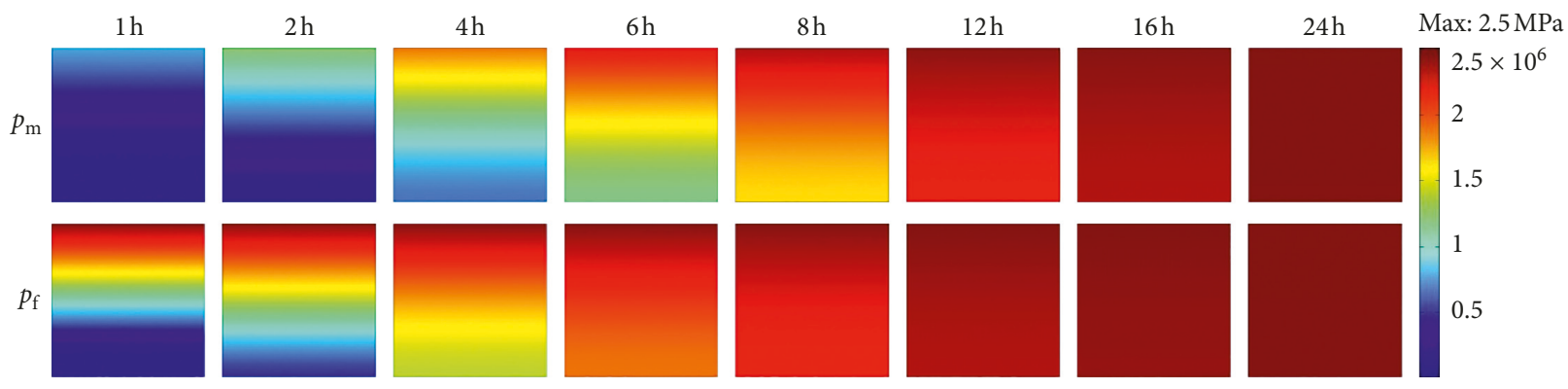

Min: $0.1 \mathrm{MPa}$

Figure 5: Gas pressure change law of the model.

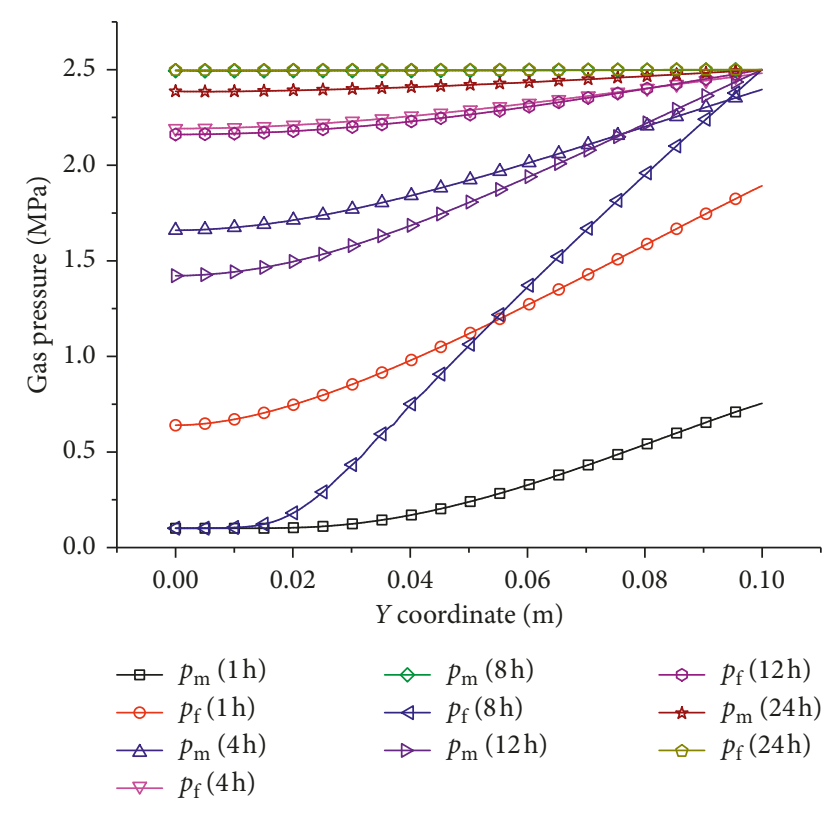

Figure 6: Gas pressure distribution along the monitoring line.

3.4. Effect of Adsorption on Permeability Distribution. Based on the dual-porous medium model, the fracture pressure and the matrix pressure are calculated through different equations. Therefore, we can further understand the dual-porous medium model by analyzing the evolution rule of permeability. Figure 7 is the variation diagram of coal permeability and gas content of coal mass at different time. The permeability of coal fractures decreases gradually. It is caused by the adsorption swelling of the matrix and the reduction of the crack opening in the coal mass.

Coal mass expends and the fracture aperture decreases when it absorbs gas. Meanwhile, the coal mass is limited by the boundary conditions, so coal deforms under the combined influence of the change of gas pressure, expansion stress, and effective stress [25]. Figure 7 shows that the permeability decreases with time, and the volumetric strain caused by adsorption is the main reason. There is an inverse relationship between the permeability and the content of gas; that is, the gas content of coal increases ceaselessly and permeability decreases gradually. The maximum of permeability ratio decreased from 0.901 to 0.876 , while the minimum of gas content increased from $0.00239 \mathrm{~m}^{3} / \mathrm{kg}$ to $0.0481 \mathrm{~m}^{3} / \mathrm{kg}$ when the time increased from $1 \mathrm{~h}$ to $8 \mathrm{~h}$. As time goes, the permeability of coal gradually stabilizes and tends to be at a uniform state.

The changes of permeability and porosity of a point are analyzed. The point is at the center of the model, and its coordinates are $(0.05 \mathrm{~m}, 0.05 \mathrm{~m})$. Figure 8 is the figure of permeability, porosity, gas content, and temperature change at the monitoring point A. Figure 8 shows that the evolution of permeability and gas content at point A are consistent with Figure 7 . The temperature and gas content increase with the increase of time. The permeability and porosity of coal decrease rapidly before $150 \mathrm{~h}$, which is mainly caused by the large pressure difference. Pore pressure and adsorption expansion affect together, which causes the change of permeability and porosity. With the decrease of pressure difference in coal mass, the change speed of permeability and porosity decreases gradually and tends to be stable finally. 


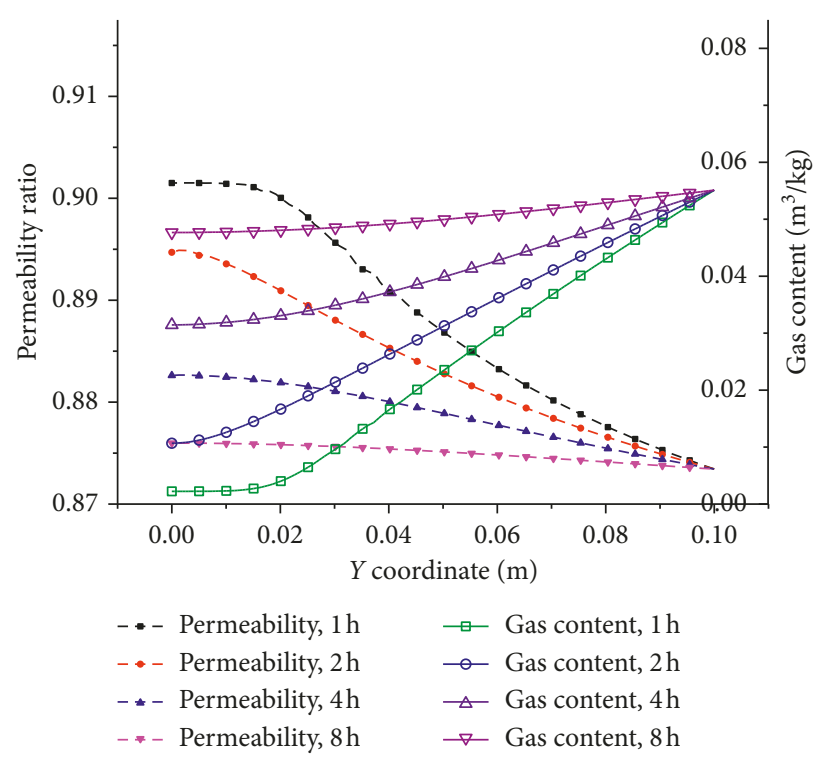

Figure 7: Permeability distribution along the monitoring line.

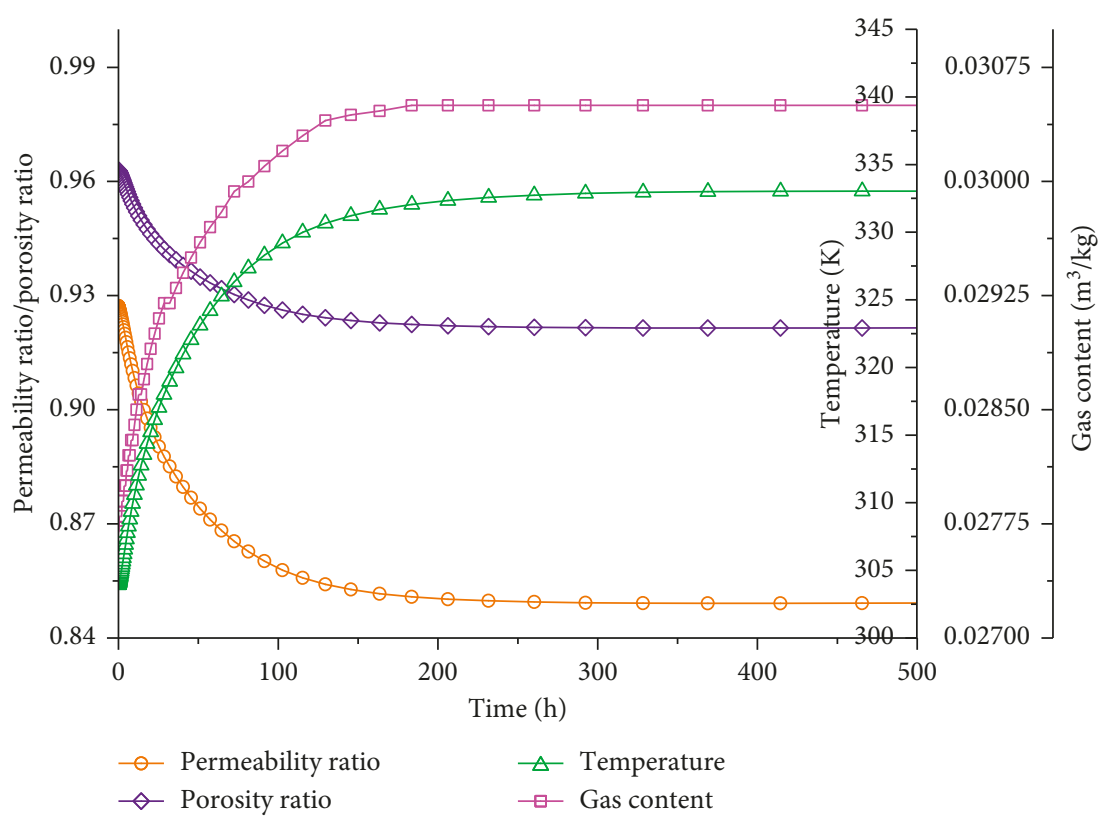

FIgURe 8: Permeability distribution of the monitoring point A.

3.5. Effect of Temperature on Permeability Distribution. Figure 9 gives the temperature change law of the model. The temperature at the entrance is relatively high, and the temperature in the coal seam keeps rising. The temperature at point $\mathrm{A}$ increases from $303.8 \mathrm{~K}$ at $1 \mathrm{~h}$ to $308.6 \mathrm{~K}$ at $8 \mathrm{~h}$, finally reaching $322.7 \mathrm{~K}$ at $40 \mathrm{~h}$. The deformation characteristics of coal seam are given in Figure 10. After the coalbed is heated up, the adsorption increases and the volume expands. The bottom, left, and right boundaries of coal seam are restricted, so there is no normal displacement in this boundaries. When the gas pressure rises on the upper boundary of the coal mass, the coal mass absorbs gas constantly, which leads to expansive deformation of coal mass and expanding outwards of the upper boundary. These characteristics also show that the volumetric strain induced by gas adsorption plays a key role. If the effect of gas adsorption is not considered, the effective stress will decrease, and the permeability of coal may increase. If the influence of gas adsorption is ignored, pore pressure may be underestimated.

The distributions of gas pressure and permeability under different temperatures are given in Figures 11 and 12. It indicates that temperature has a greater impact on the gas migration in the coal seam. The influence of temperature is reduced as the coal seam gas pressure approaches the setting pressure. High temperature can promote the desorption of gas and then change the fracture aperture and porosity of coal mass. The influence of temperature on the porosity of coal 
$1 \mathrm{~h}$

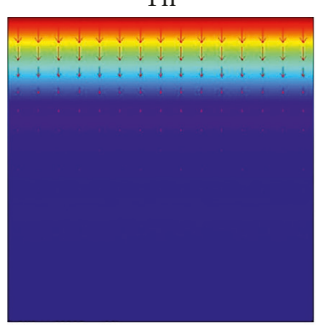

$40 \mathrm{~h}$

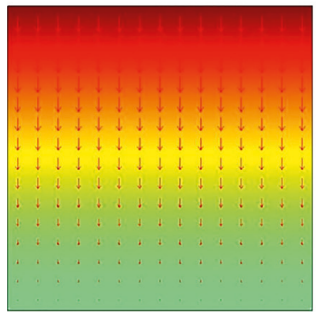

$4 \mathrm{~h}$

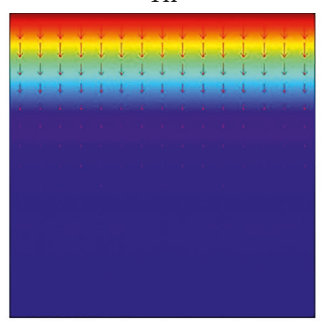

$120 \mathrm{~h}$

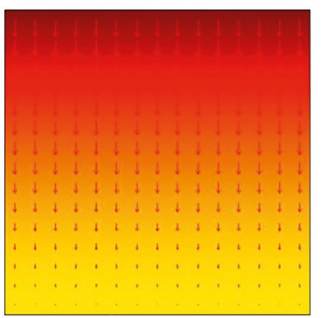

$8 \mathrm{~h}$

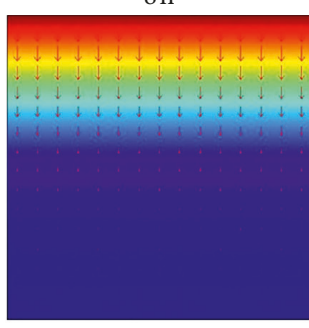

$200 \mathrm{~h}$

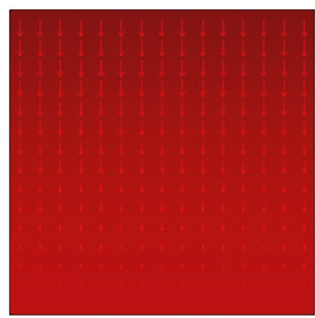

$24 \mathrm{~h}$

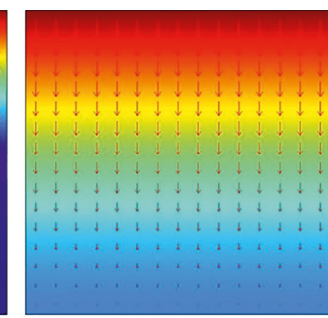

$300 \mathrm{~h}$

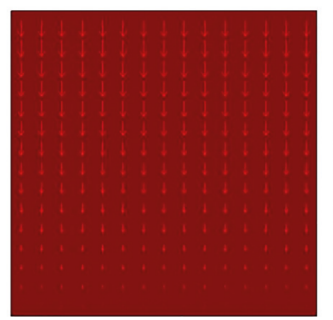

Max: 333 K

330

325

320

315

310

305

Min: $303 \mathrm{~K}$

FIgURE 9: Temperature change law of the model.

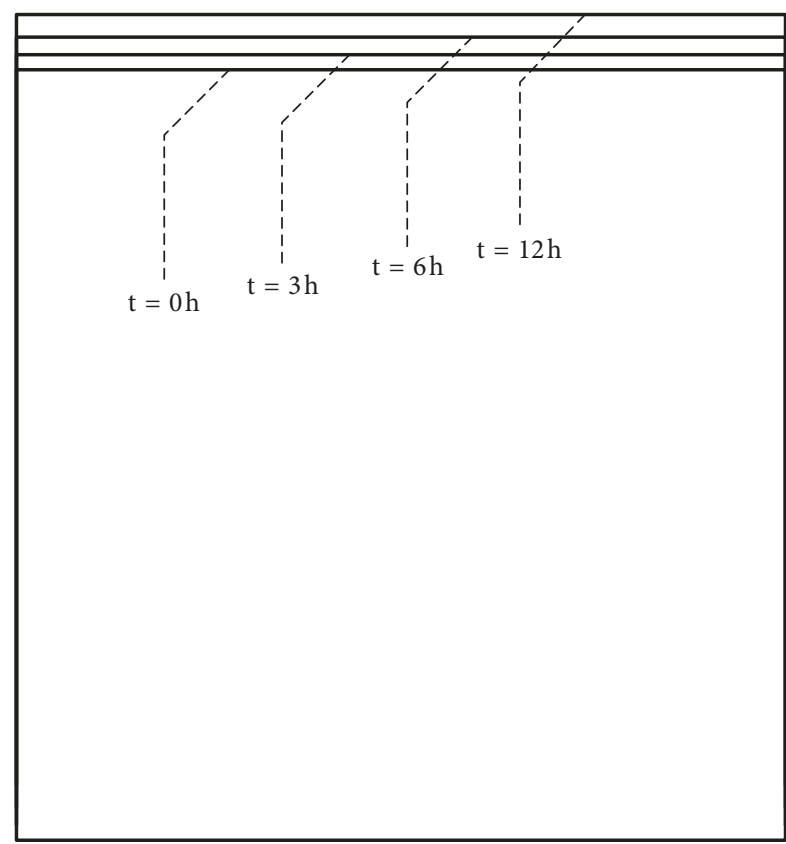

FIGURE 10: Evolution of the coal volume with time under the effect of temperature.

seam is a complicated process. The temperature evolution is obtained under the coupling effect of multiple factors, such as restriction on the boundary condition and adsorptive expansion deformation of coal mass. At the initial stage, the gas pressure and porosity of coal mass differ greatly at different temperatures. Gas pressure and permeability change constantly with the increase of time, and finally attain states of equilibrium. The gas pressure of point $\mathrm{A}$ is $1.05 \mathrm{MPa}$, 1.27 MPa, and $1.41 \mathrm{MPa}$ at $1 \mathrm{~h}$ when the initial boundary temperature is $333 \mathrm{~K}, 353 \mathrm{~K}$, and $373 \mathrm{~K}$, respectively.

In order to quantify the influence of gas diffusion behavior, the fracture pressure distribution without Klinkenberg

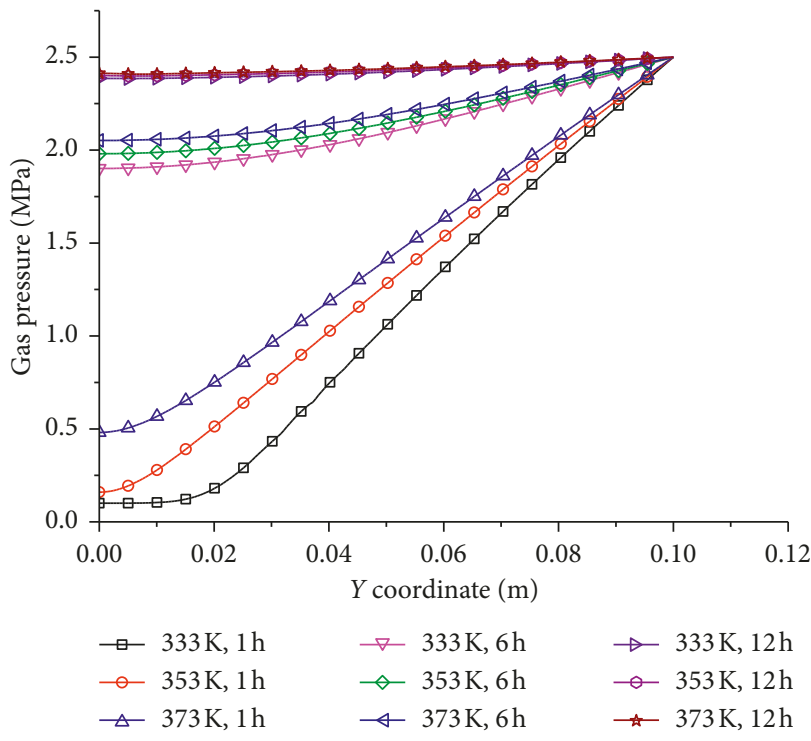

Figure 11: Gas pressure distribution with different temperatures.

effect and different adsorption times was also plotted as shown in Figure 13. The adsorption time significantly affects the distribution of fracture pressure. When the adsorption time is large, the gas pressure of coal seam will be underestimated. The influence of diffusion effect is the same as that of Klinkenberg effect at the same order of magnitude. In the sorption stage, the total volume expansion effect and Klinkenberg effect of coal increase the permeability. When the Klinkenberg effect is not considered, the adsorption pressure will be underestimated.

\section{Conclusions}

A new model about heat transfer was developed to study the dynamic problem of gas adsorption in coal seam under 


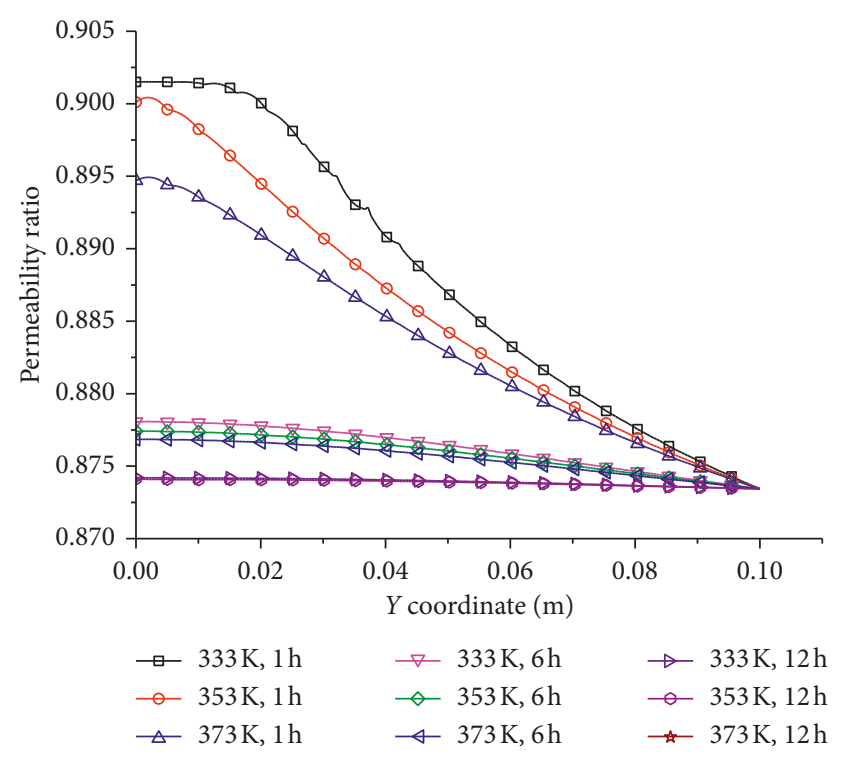

Figure 12: Permeability distribution with different temperatures.

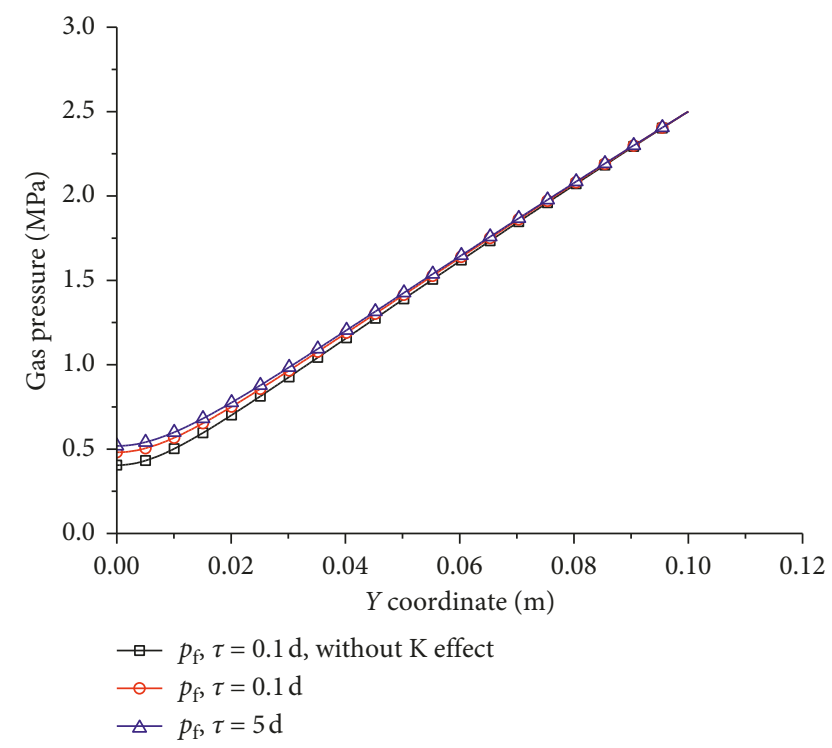

FIGURE 13: Gas pressure distribution under different cases.

variable temperature conditions. In the model, the coal mass was regarded as a dual-porous medium, and each point of the coal mass has two different pressures, i.e., pore pressure and matrix pressure. The relationship between adsorption equilibrium and permeability evolution was analyzed through the numerical method. The main results are as follows:

The gas pressure in fractures is generally higher than that in the matrix blocks in the calculation model. The matrix also continues to adsorb gas, and the pressure rises gradually. Finally, pore pressure and matrix pressure reach the equilibrium state.

The adsorption of coal increases with the increase of gas pressure, and the gas content increases continuously. The porosity of coal mass decreases with the increase of time, while the temperature and gas content increase with the increase of time.

The higher temperature induces the faster increase of the gas pressure. Temperature has a greater impact on the gas migration. As the coal seam gas pressure approaches the setting pressure, the influence of temperature is reduced.

\section{Data Availability}

The data used to support the findings of this study are included within the article.

\section{Conflicts of Interest}

The authors declare that they have no conflicts of interest.

\section{Acknowledgments}

This work was supported by the China Postdoctoral Science Foundation (Grant no. 2018M633549), Fundamental Research Funds for the Central Universities (Grant no. 2015XKZD06), and National Natural Science Foundation of China (Grant nos. 51674247 and 51804302).

\section{References}

[1] Y. Xue, Z. Cao, F. Du, and L. Zhu, "The influence of the backfilling roadway driving sequence on the rockburst risk of a coal pillar based on an energy density criterion," Sustainability, vol. 10, no. 8, p. 2609, 2018.

[2] Y. Zhang, J. Underschultz, L. Langhi, D. Mallants, and J. Strand, "Numerical modelling of coal seam depressurization during coal seam gas production and its effect on the geomechanical stability of faults and coal beds," International Journal of Coal Geology, vol. 195, pp. 1-13, 2018.

[3] G. Hu, N. Yang, G. Xu, and J. Xu, "Experimental investigation on variation of physical properties of coal samples subjected to microwave irradiation," Journal of Applied Geophysics, vol. 150, 2018.

[4] Z. Cao, Y. Zhou, P. Xu, and J. Li, "Mechanical response analysis and safety assessment of shallow-buried pipeline under the influence of mining," CMES: Computer Modeling in Engineering and Sciences, vol. 101, no. 5, pp. 351-364, 2014.

[5] Y. Xue, T. Teng, L. Zhu et al., "Evaluation of the non-Darcy effect of water inrush from karst collapse columns by means of a nonlinear flow model," Water, vol. 10, no. 9, p. 1234, 2018.

[6] A. Zhou, K. Wang, L. Wu, and Y. Xiao, "Influence of gas ventilation pressure on the stability of airways airflow," International Journal of Mining Science and Technology, vol. 28, no. 2, pp. 297-301, 2018.

[7] G. Hu, J. Xu, T. Ren, Y. Dong, W. Qin, and Z. Shan, "Field investigation of using water injection through inseam gas drainage boreholes to control coal dust from the longwall face during the influence of abutment pressure," International Journal of Surface Mining Reclamation and Environment, vol. 30, no. 1, pp. 48-63, 2016.

[8] Y. Xue, F. Dang, Z. Cao et al., "Numerical analysis of heat and gas transfer characteristics during heat injection processes based on a thermo-hydro-mechanical model," Energies, vol. 11, no. 7, p. 1722, 2018.

[9] D. Wang, R. Lv, J. Wei, P. Zhang, C. Yu, and B. Yao, “An experimental study of the anisotropic permeability rule of coal 
containing gas," Journal of Natural Gas Science and Engineering, vol. 53, pp. 67-73, 2018.

[10] G. Wang, L. Dou, C. Wu et al., "Unstable energy triggering mechanism of rock burst," Journal of China University of Mining and Technology, vol. 47, pp. 190-196, 2018.

[11] D. K. Wang, S. M. Liu, J. P. Wei, H. L. Wang, and B. H. Yao, "A research study of the intra-nanopore methane flow law," International Journal of Hydrogen Energy, vol. 42, no. 29, pp. 18607-18613, 2017.

[12] J. Noorishad, C. F. Tsang, and P. A. Witherspoon, "Coupled thermal-hydraulic-mechanical phenomena in saturated fractured porous rocks: numerical approach," Journal of Geophysical Research: Solid Earth, vol. 89, no. 12, pp. 10365-10373, 1984.

[13] D. F. Mctigue, "Thermoelastic response of fluid-saturated porous rock," Journal of Geophysical Research Solid Earth, vol. 91, no. 9, pp. 9533-9542, 1986.

[14] S. Harpalani and R. A. Schraufnagel, "Shrinkage of coal matrix with release of gas and its impact on permeability of coal," Fuel, vol. 69, no. 5, pp. 551-556, 1990.

[15] Y. Zhou, R. K. N. D. Rajapakse, and J. Graham, "A coupled thermoporoelastic model with thermo-osmosis and thermalfiltration," International Journal of Solids and Structures, vol. 35, no. 34, pp. 4659-4683, 1998.

[16] W. C. Zhu, C. H. Wei, J. Liu, H. Y. Qu, and D. Elsworth, "A model of coal-gas interaction under variable temperatures," International Journal of Coal Geology, vol. 86, no. 2-3, pp. 213-221, 2011.

[17] Y. Cai, D. Liu, and Z. Pan, "Partial coal pyrolysis and its implication to enhance coalbed methane recovery: a simulation study," Energy and Fuels, vol. 31, no. 5, pp. 4895-4903, 2017.

[18] H. Qu, J. Liu, Z. Pan, Y. Peng, and F. Zhou, "Simulation of coal permeability under non-isothermal $\mathrm{CO} 2$ injection," International Journal of Oil, Gas and Coal Technology, vol. 15, no. 2, pp. 190-215, 2017.

[19] Z. Junxiang, L. Bo, and S. Yuning, "Dynamic leakage mechanism of gas drainage borehole and engineering application," International Journal of Mining Science and Technology, vol. 28, no. 3, pp. 505-512, 2018.

[20] D. J. Remner, T. Ertekin, W. Sung, and G. R. King, "A parametric study of the effects of coal seam properties on gas drainage efficiency," SPE Reservoir Engineering, vol. 1, no. 6, pp. 633-646, 1986.

[21] L. J. Klinkenberg, The Permeability of Porous Media to Liquids and Gases, Drilling and Production Practice, American Petroleum Institute, Washington, DC, USA, 1941.

[22] H. Zhang, J. Liu, and D. Elsworth, "How sorption-induced matrix deformation affects gas flow in coal seams: a new FE model," International Journal of Rock Mechanics and Mining Sciences, vol. 45, no. 8, pp. 1226-1236, 2008.

[23] F. Tong, L. Jing, and R. W. Zimmerman, "A fully coupled thermo-hydro-mechanical model for simulating multiphase flow, deformation and heat transfer in buffer material and rock masses," International Journal of Rock Mechanics and Mining Sciences, vol. 47, no. 2, pp. 205-217, 2010.

[24] C. A. Mora and R. A. Wattenbarger, "Comparison of computation methods for CBM performance," Journal of Canadian Petroleum Technology, vol. 48, no. 4, pp. 42-48, 2009.

[25] Z. Li, X. He, and L. Dou, "Control measures and practice for rock burst induced by overburden fracture in top-coal caving mining," Journal of China University of Mining and Technology, vol. 47, pp. 162-171, 2018. 


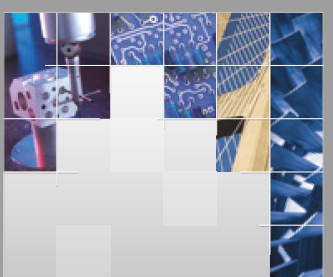

\section{Enfincering}
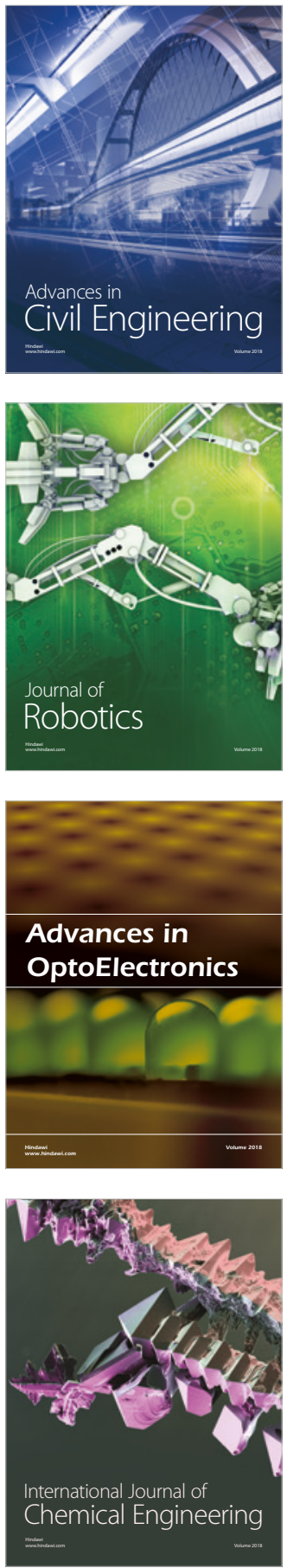

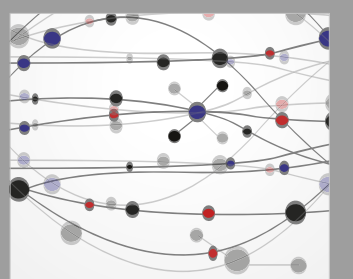

\section{Rotating \\ Machinery}

The Scientific World Journal

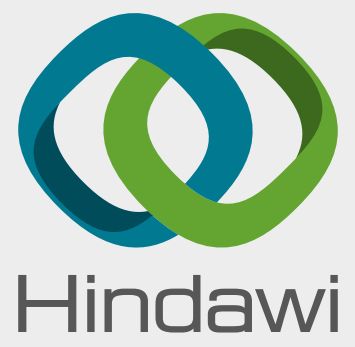

Submit your manuscripts at

www.hindawi.com
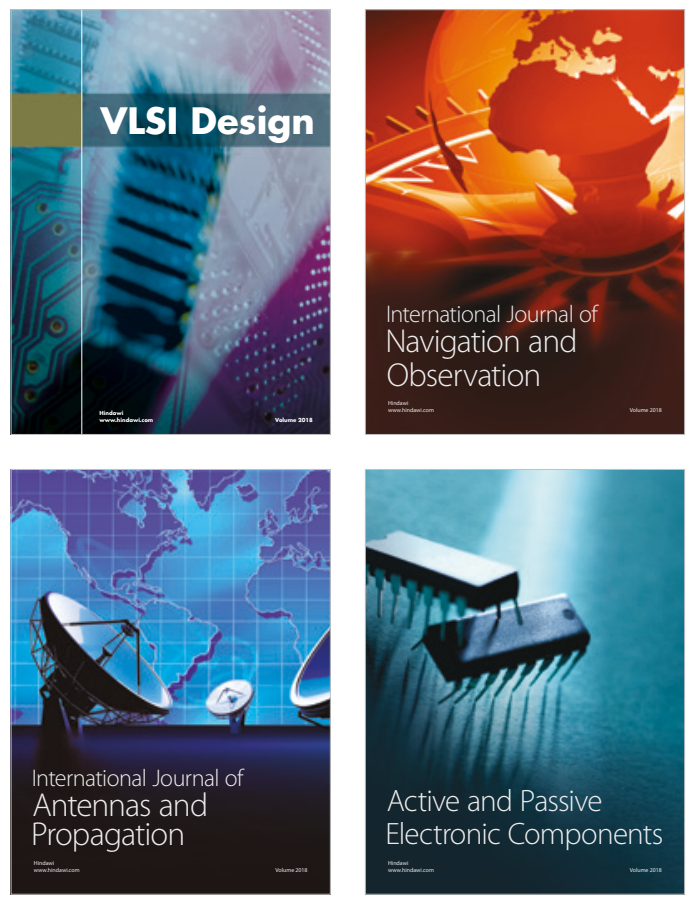
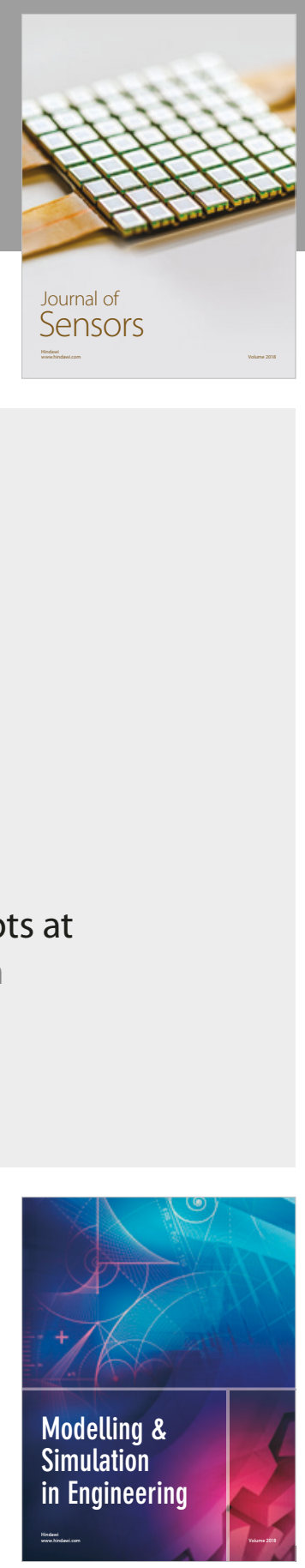

\section{Advances \\ Multimedia}
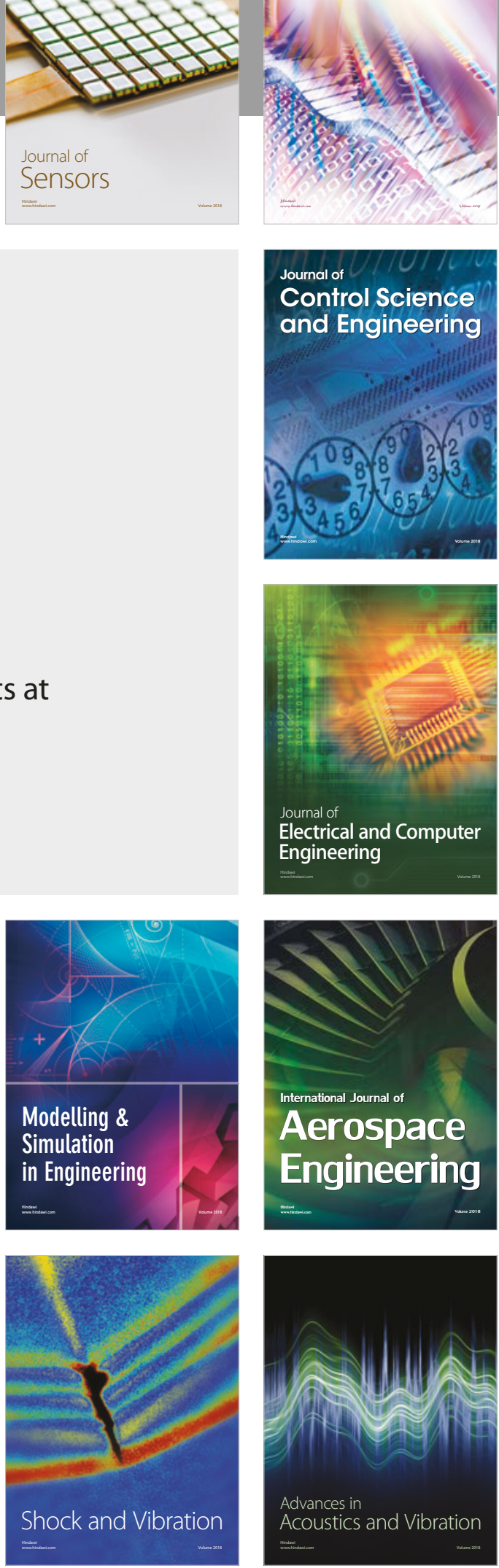